\title{
Significance of gastrin-releasing peptide in ovarian cancer ES2 cells
}

\author{
YANYAN JIA ${ }^{1}$, HUIRONG SHI ${ }^{1}$ and DONGMEI FAN ${ }^{2}$ \\ ${ }^{1}$ Department of Gynecology, The First Affiliated Hospital, Zhengzhou University, Zhengzhou, Henan 450052; \\ ${ }^{2}$ Department of Gynecology, The First Affiliated Hospital, Henan Scientific and Technologic University, \\ Luoyang, Henan 471003, P.R. China
}

Received June 25, 2014; Accepted March 4, 2015

DOI: $10.3892 / \mathrm{ol} .2015 .3240$

\begin{abstract}
The present study aimed to investigate the effect of gastrin-releasing peptide (GRP) on the proliferation and invasion of ovarian cancer ES2 cells. The ovarian cancer ES2 cells were transfected with small interfering RNA against GRP. Cell proliferation was assessed using the Trypan blue assay, apoptosis was determined using propidium iodide/fluorescein isothiocyanate and flow cytometry, and the invasion ability was detected using the Transwell assay. The results revealed that the expression of GRP significantly decreased following transfection with GRP-short hairpin RNA. Furthermore, the silencing of GRP resulted in increased apoptosis and a reduced invasive ability of the ES2 cells. It was concluded that GRP may regulate the proliferation and migration of human ovarian cancer cells, which indicates that GRP may be a potential novel target for the treatment of ovarian cancer.
\end{abstract}

\section{Introduction}

Gastrin-releasing peptide (GRP) is a 27-amino acid peptide, which was first identified as a cytokine secreted from P cells in the alimentary tract. The local secretion of GRP regulates gastrointestinal hormones and gallbladder contraction (1). Previous studies revealed that GRP and the GRP receptor (GRPR) were overexpressed not only in the gastrointestinal tract, but also in a number of other malignant tumors and cell lines (2-5). It has been established that epithelial cells from regions other than the parenteral tract can also excessively secret GRP under the regulation of carcinogenic factors. GRP can then bind to the GRPR and promote the mitosis of cells.

We previously screened samples of serum, ascites and cyst fluid from 30 ovarian carcinoma patients by matrix-assisted

Correspondence to: Miss. Yanyan Jia, Department of Gynecology, The First Affiliated Hospital, Zhengzhou University, 1 Jianshe Road, Zhengzhou, Henan 450052, P.R. China

E-mail: jiayy_2014@163.com

Key words: gastrin-releasing peptide, small interfering RNA, ovarian cancer, cell proliferation laser desorption/ionization time-of-flight mass spectrometer technology combined with WCX magnetic beads. The results indicated that the elevated peptide peaks at 2881 and $2897 \mathrm{Da}$ may correspond to the same GRP polypeptide, and confirmed that GRP may be involved in the carcinogenesis of ovarian cancer (6). The biological effects of GRP in colorectal, gastric and prostate cancer have been previously reported (7-9). However, studies concerning the biological effects of GRP in ovarian cancer have yet to be conducted.

In the present study, a small interfering RNA (siRNA) against GRP was constructed (10) and transfected into human ovarian cancer ES2 cells, which are known to highly express GRP. The biological changes in the ES2 cells were then investigated.

\section{Materials and methods}

Materials. The Escherichia coli (E. coli; DH5 $\alpha)$ and pGenesil-1.1dBm2 vector were purchased from the Wuhan JinSai Biological Technology Engineering Company (Wuhan, China). Monoclonal mouse anti-human GRP (dilution, 1:1,000) and mouse anti-human $\beta$-actin (dilution: 1:1,000) antibodies were obtained from R\&D Systems, Inc., (Minneapolis, MN, USA). The human ovarian cancer ES2 cell line was supplied by the China Center for Type Culture Collection at Wuhan University (Wuhan, China).

Cell culture. The ES2 cells were cultured in RPMI-1640 medium supplemented with $10 \%$ fetal bovine serum, $100 \mu \mathrm{g} / \mathrm{ml}$ streptomycin and $100 \mathrm{U} / \mathrm{ml}$ penicillin. The cell cultures were maintained in a humidified atmosphere of $5 \% \mathrm{CO}_{2}$ at $37^{\circ} \mathrm{C}$ in an incubator.

Design of short hairpin (sh)RNA construction for GRP. According to the GRP complementary (c)DNA sequence obtained from Genbank, the GRP-targeted shRNA construct was designed using the RNAi software from Ambion Life Technologies (Carlsbad, CA, USA). The cDNA target sequence was $192-210 \mathrm{bp}$ in length. The oligonucleotide was synthesized by Wuhan JinSai Biological Technology Engineering Company, and the sequence of the GRP-shRNA was as follows (the guide peptide is in bold): Sense, 5'-CACCACCGTGCTGACCAAGATGTTTCA 
AGACGACATCTTGGTCAGCACGGTTTTTTTG-3'; and antisense, 5'-AGCTCAAAAAAACCGTGCTGACCAAGA TGTCGTCTTGAAACATCTTGGTCAGCACGGT-3'.

Construction and cloning of pGenesil-GRP-shRNA. The GRP-shRNA was inserted into the SacI site of the expression vector, pGenesil-1.1dBm2. The product of the recombination reactions, pGenesil-GRP-shRNA, was used to transform the competent $E$. coli strain, DH5 $\alpha$, during the transformation assay. Briefly, the bacteria were washed with a $0-100 \mathrm{mM} \mathrm{CaCl}_{2}$ solution, resuspended in $200 \mu \mathrm{l}$ of the same solution, and then placed on ice for $1 \mathrm{~h}$. Next, the pGenesil-GRP-shRNA plasmid DNA was added for $30 \mathrm{~min}$. The sample was then incubated at $42^{\circ} \mathrm{C}$ for $90 \mathrm{sec}$. Following the addition of $1 \mathrm{ml}$ Luria-Bertani broth, the sample was incubated for $1 \mathrm{~h}$ at $37^{\circ} \mathrm{C}$. In total, $100 \mu \mathrm{l}$ of the transformation mixture was transferred onto a Luria-Bertani agar plate containing $100 \mu \mathrm{g} / \mathrm{ml}$ kanamycin and $100 \mu \mathrm{g} / \mathrm{ml}$ neomycin. The transformation frequency was determined by calculating the ratio of the number of transformants per viable cell per milliliter.

The plate was then cultured at $37^{\circ} \mathrm{C}$ for $16-24 \mathrm{~h}$, the positive colonies were amplified in Luria-Bertani broth and the plasmid was extracted using a plasmid extraction kit (Qiagen $\mathrm{GmbH}$, Hilden, Germany) The plasmid was identified by electrophoresis and restriction enzyme digestion.

pGenesil-GRP-shRNA transfection. An equal number of ES2 cells $\left(0.8 \times 10^{6}\right.$ per well $)$ were seeded into $35-\mathrm{mm}$ dishes. The cells were left for $24 \mathrm{~h}$ to attach and reach $70-80 \%$ confluency. The transfection assay was performed using Lipofectamine 2000 transfection reagent (Invitrogen Life Technologies, Carlsbad, CA, USA) according to the manufacturer's instructions. Untransfected cells and cells transfected with an empty vector were used as the controls.

The efficiency was assessed according to the concentration of GFP at $24 \mathrm{~h}$ post-transfection. The cells were harvested 2-3 days after transfection, and then cultured in complete medium containing $800 \mu \mathrm{g} / \mathrm{ml} \mathrm{G} 418$ for 7-8 days. Fresh medium was added every 3 days until the untransfected control cells were all dead. Next, the cells were cultured in complete medium containing $200 \mu \mathrm{g} / \mathrm{ml} \mathrm{G} 418$. The positive clones appeared $\sim 20$ days later. These positive cells were recultured in fresh bottles and designated pGenesil-GRP-shRNA cells or pGenesil-1.1dBm2 cells.

Reverse transcription polymerase chain reaction (RT-PCR). The total RNA was extracted from the positive ES2 cell clones using TRIzol (Invitrogen Life Technologies), and then reversed into cDNA by the Super SCRIPTM cDNA kit from Takara Biotechnology Co., Ltd. (Dalian, China). In order to examine the expression of endogenous GRP mRNA, the RT-PCR was performed using the following primers: GRP forward, 5'-AGAGTACATCAGGTGGGA-3' and reverse, 5'-CAGAAG ATGCTGCTTTAAAA-3' (product size, 375 bp); and $\beta$-actin forward, 5'-ACTCTTCCAGCCTTCCTTC-3' and reverse, 5'-AATCCTGAGTCAAGCCAAA-3' (product size, $490 \mathrm{bp}$ ). $\beta$-actin was used as the internal reference. The amplification conditions were as follows: $94^{\circ} \mathrm{C}$ for $5 \mathrm{~min}$, followed by 30 cycles at $94^{\circ} \mathrm{C}$ for $40 \mathrm{sec}, 60^{\circ} \mathrm{C}$ for $30 \mathrm{sec}$ and $72^{\circ} \mathrm{C}$ for $30 \mathrm{sec}$, and finally $72^{\circ} \mathrm{C}$ for $5 \mathrm{~min}$. The PCR products were electrophoresed in 1.5\% agarose gel, stained with ethidium bromide and then analyzed using the Q550IW image analysis system (Leica Imaging Systems, Cambridge, UK). The integral optical density of each band was calculated according to the following equation: Integral absorbance $=$ average absorbance $\mathrm{x}$ area.

Western blotting. The positive ES2 cell clones were resuspended in SDS loading buffer, heated for $10 \mathrm{~min}$ at $100^{\circ} \mathrm{C}$, and then centrifuged at $13,000 \mathrm{x}$ g for $15 \mathrm{~min}$ at $4^{\circ} \mathrm{C}$. The supernatants were collected and separated by SDS-PAGE electrophoresis, and then transferred to a polyvinylidene difluoride membrane. The membrane was dipped in 5\% skimmed milk powder for $2 \mathrm{~h}$ in order to block non-specific binding sites, and then incubated with GRP or $\beta$-actin primary antibodies at $4^{\circ} \mathrm{C}$ overnight. Subsequent to rinsing three times in Tris-buffered saline and Tween 20 (TBST), the membrane was incubated with the corresponding secondary antibody labeled with horseradish peroxidase for $2 \mathrm{~h}$, and then rinsed in TBST a further three times. Next, the membrane was incubated with SuperSignal West Dura Chemiluminescent Substrate (Thermo Fisher Scientific) for $5 \mathrm{~min}$. Finally the images were obtained by X-ray film exposure.

Cell proliferation detection. The untransfected cells and the ES2 cells transfected with pGenesil-GRP-shRNA or pGenesil-1.1dBm2 vector were cultured. The percentage of dead cells was determined by staining with Trypan blue each day for 7 days.

Flow cytometric analysis of apoptosis. The ES2 cells were collected and washed once with phosphate-buffered saline and then resuspended in $1 \times 10^{6} / \mathrm{ml}$ annexin-binding buffer. In total, $100 \mu \mathrm{l}$ of the cell solution was stained with $5 \mu \mathrm{l}$ Annexin $\mathrm{V}$ and $5 \mu \mathrm{l}$ propidium iodide for $15 \mathrm{~min}$. The stained cells were analyzed by FACScan flow cytometry (BD Biosciences, Franklin Lakes, NJ).

Cell invasion. The Transwell assay was performed using a commercial kit purchased from Corning Inc. (Corning, New York, NY, USA), as previously described (11). Briefly, collagen-coated membranes were seeded with $1 \times 10^{5}$ cells in $500 \mu 1$ DMEM. In all instances, cell migration was assessed $5 \mathrm{~h}$ after plating, as recommended by the manufacturer.

Statistical analysis. The data are expressed as the mean \pm standard deviation and were analyzed using SPSS 13.0 (SPSS, Inc., Chicago, IL, USA). Student's t-test was performed for comparison between two groups. $\mathrm{P}<0.05$ was used to indicate a statistically significant difference.

\section{Results}

Successful construction and transfection of pGenesil-GRP-shRNA. A SacI restriction site was designed at each end of the GRP-shRNA fragment. The fragment was inserted into the vector, pGenesil-1.1 $\mathrm{dBm} 2$, which was digested by SacI. As shown in Fig. 1, when the pGenesil-GRP-shRNA plasmid was digested by SacI, a 916-bp band appeared, which 


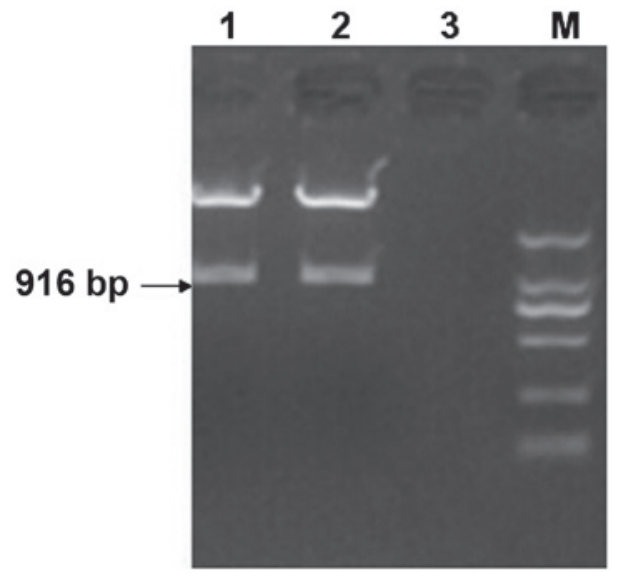

Figure 1. Digestion of pGenesil-GRP-shRNA by SacI. Lanes 1 and 2 , pGenesil-GRP-shRNA; lane 3, negative control; M, DNA marker with 2,000, $1,000,750,500,250$ and 100-bp bands (top to bottom). GRP, gastrin-releasing peptide; shRNA, short hairpin RNA.

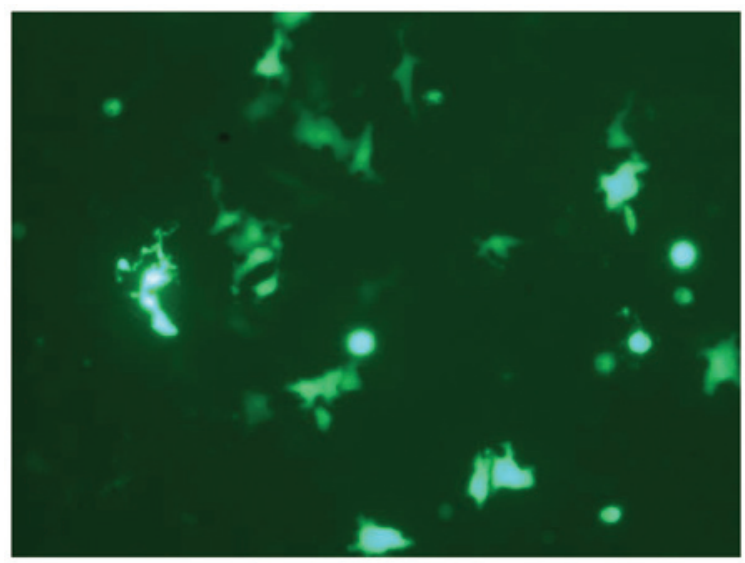

Figure 2. Green fluorescent protein in ES2 cells transfected with pGenesil-GRP-shRNA. GRP, gastrin-releasing peptide; shRNA, short hairpin RNA.

corresponded to GRP-shRNA. The pGenesil-GRP-shRNA plasmid was sequenced, and it was confirmed that pGenesil-GRP-shRNA had been successfully constructed.

For the transfection assays, the cells were screened using the G418 antibiotic; only the successfully transfected cells were able to survive. The pGenesil-1.1dBm2 vector carried a green fluorescent protein, which emitted green fluorescence under light excitation. As shown in Fig. 2, the positive clones of ES2 appeared green under the laser.

GRP silencing by GRP-shRNA. As shown in Fig. 3, the expression level of GRP was calculated by the integral optical density of the band. The mRNA level of GRP in the transfected cells was significantly lower than that of the control transfected and untransfected groups $(0.36,0.87$ and 0.91 , respectively; $\mathrm{P}<0.05)$. The protein level of GRP was also markedly decreased in the transfected group compared with the control transfected and untransfected groups $(0.13,0.47$ and 0.49 , respectively; $\mathrm{P}<0.05$; Fig. 4). The results revealed that the ES2 cells were successfully transfected by pGenesil-GRP-shRNA and that the expression of GRP was significantly silenced.

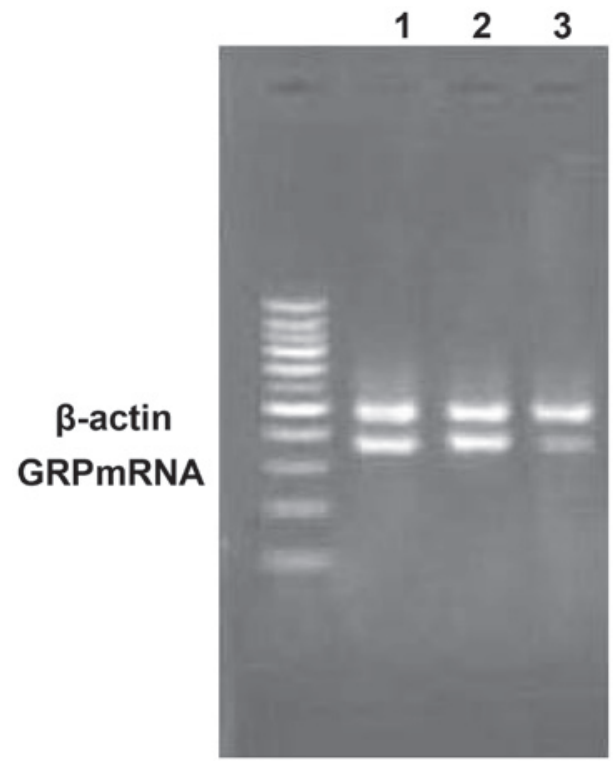

Figure 3. Expression of GRP mRNA following silencing. Lane Ma,100bp Plus II DNA marker with 1,500, 1,000, 900, 800, 700, 500, 400, 300, 200 and 100-bp bands (top to bottom) Lane 1, untransfected group; lane 2, empty vector-transfected group; lane 3, GRP-shRNA-transfected group. GRP, gastrin-releasing peptide; shRNA, short hairpin RNA.

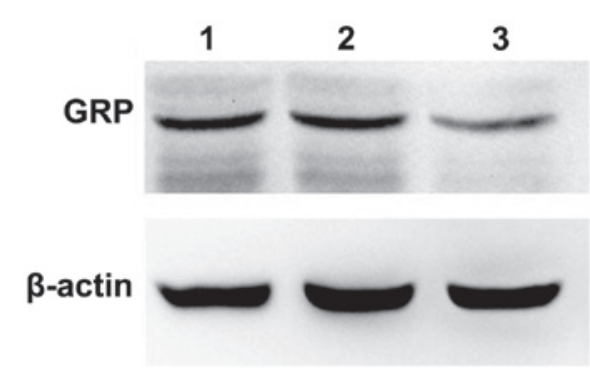

Figure 4. Protein levels of GRP following silencing. Lane 1, untransfected group; lane 2, empty vector-transfected group;lane 3, GRP-shRNA-transfected group. GRP, gastrin-releasing peptide; shRNA, short hairpin RNA.

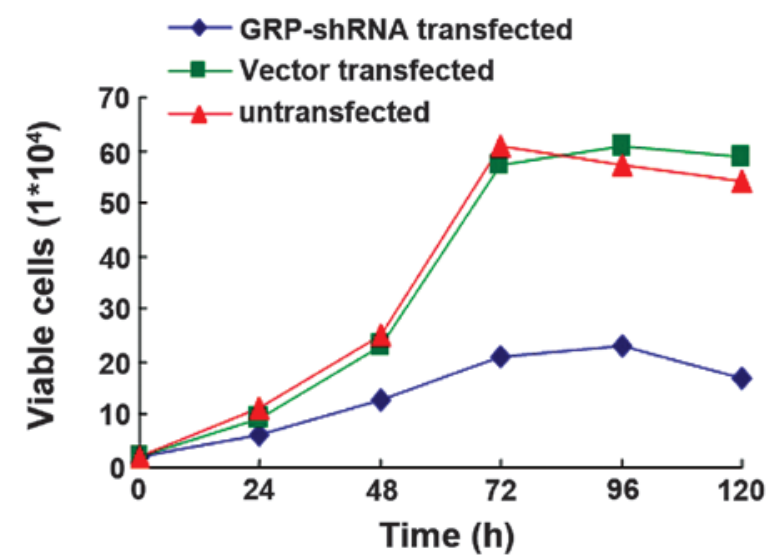

Figure 5. GRP silencing-induced cell proliferation inhibition. GRP, gastrin-releasing peptide; shRNA, short hairpin RNA.

GRP silencing inhibits proliferation. The ES2 cells were transfected with GRP-shRNA or vector alone, untransfected cells were used as the control. The cells were then cultured for 
A

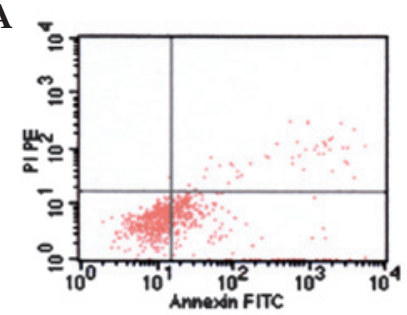

B

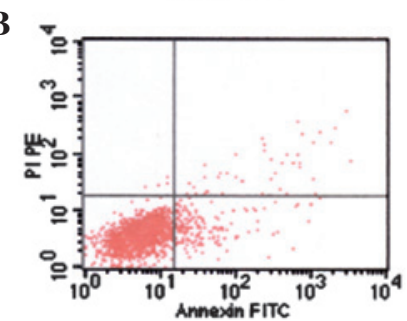

C

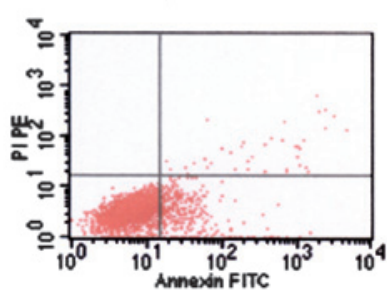

Acquisition Date: 27-Aug-9 Gate: G1

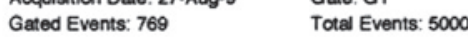

\begin{tabular}{crrr} 
Quad & Events & $\%$ Gated & $\%$ Total \\
\hline UL. & 2 & 0.26 & 0.04
\end{tabular}

$\begin{array}{llll}\text { UR } & 45 & 5.85 & 0.90\end{array}$

L $\quad 480 \quad 62.42 \quad 9.60$

$31.47 \quad 4.84$

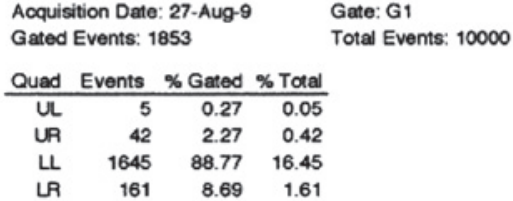

Acquisition Date: 27-Aug-9 Gate: 61 Gated Events: $2486 \quad$ Total Events: 10000

Figure 6. GRP silencing-induced apoptosis. (A) GRP-shRNA-transfected group, (B) vector-transfected group and (C) untransfected group. GRP, gastrin-releasing peptide; shRNA, short hairpin RNA; FITC, fluorescein isothiocyanate; PI, propidium iodide; PE, phycoerythrin.
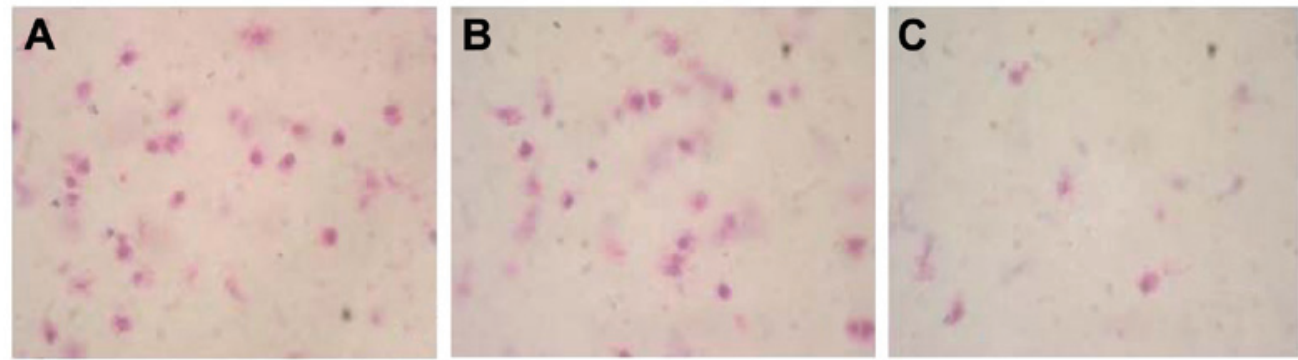

Figure 7. Invasion ability of ES2 cells following GRP silencing. (A) Untransfected group, (B) vector-transfected group and (C) GRP-shRNA-transfected group. GRP, gastrin-releasing peptide; shRNA, short hairpin RNA.

5 days and the cell viability was detected each day. The results demonstrated that the ES2 cells transfected with GRP-shRNA had a significantly lower proliferation ability compared with the control $(\mathrm{P}<0.05$, Fig. 5), while there was no observed difference in the proliferation ability of the ES2 cells transfected with the vector alone compared with the control $(\mathrm{P}>0.05)$ (Fig. 5).

GRP silencing induces apoptosis. As shown in Fig. 6, following transfection with pGenesil-GRP-shRNA, the apoptosis rate of the ES2 cells significantly increased (from 8.69 to 31.47\%), while that of the vector group increased only slightly (from 8.69 to $11.47 \%$ ); a significant difference was observed between the two groups $(\mathrm{P}<0.05)$.

Effects of 3.6 GRP siRNA on the invasion ability of ES2 cells. Cells that had migrated over the microporous membrane were stained by methylene blue and were counted under an inverted microscope (IX71; Olympus Corporation, Tokyo, Japan) (Fig. 7). The invasive ability of the cells (the number of cells that had migrated over the microporous membrane) in the transfected group was $21 \pm 5.3$, which was significantly lower than that of the control groups $(49 \pm 11.8$ for the vector transfected group; $43 \pm 10.1$ for the untransfected group) $(\mathrm{P}<0.05)$. The results suggested that silencing of GRP significantly weakened the invasive ability of ES2 cells, and revealed that the GRP may be important for the invasion of ovarian cancer.

\section{Discussion}

GRP and its receptor, GRPR, are overexpressed in a number of different tumors, and are believed to have an important role in carcinogenesis and tumor progression (12). It has been hypothesized that the carcinogenic ability of GRP relies on its affinity to GRPR. It was previously reported that in neurofibromatosis, silencing of GRPR through specific siRNA resulted in a significant reduction in proliferation and cell migration, and was accompanied by the inactivation of Akt and the activation of phosphatase and tensin homolog, which is a known inhibitor 
of the phosphoinositide 3-kinase/Akt (PI3K/Akt) pathway. This indicated that the PI3K/Akt signaling pathway may be regulated by GRP/GRPR (13). Another study concerning small cell lung cancer indicated that the overexpression of GRP led to an elevated level of Akt, which is known to be the initiating factor of the epidermal growth factor receptor (EGFR) signaling pathway (14). As a high level of EGFR is considered to contribute to the resistance of small lung cancer to gemcitabine, the results of the present study indicate that GRP may also have a role in chemotherapy resistance through activating the EGFR signaling pathway in tumors.

In the present study, the expression of GRP in the ES2 cells was downregulated using siRNA technology. The results revealed that the protein level of GRP significantly decreased following transfection, which was accompanied by a decrease in the proliferation and migration ability of ES2 cells, and a marked increase in the rate of apoptosis. This suggested that GRP has a role in the proliferation and cell migration of human ovarian cancer cells. This finding is consistent with that of previous studies concerning other types of tumor (15-18). Ischia et al (15) reported that GRP and GRPR are overexpressed in prostate cancer and can stimulate the growth of prostate cancer cells. In addition, GRP was found to be associated with the migration and metastasis of cancer cells in neuroblastoma and breast cancer (16-18). It can therefore be concluded that GRP may be a potential novel target for the treatment of ovarian cancers.

\section{References}

1. Roesler R, Kapczinski F, Quevedo J, et al: The gastrin-releasing peptide receptor as a therapeutic target in central nervous system disorders. Recent Pat CNS Drug Discov 2: 125-129, 2007.

2. Fang J, Lu Y, Ouyang K, et al: Specific antibodies elicited by a novel DNA vaccine targeting gastrin-releasing peptide inhibit murine melanoma growth in vivo. Clin Vaccine Immunol 16: 1033-1039, 2009.

3. Qiao J, Kang J, Ishola TA, et al: Gastrin-releasing peptide receptor silencing suppresses the tumorigenesis and metastatic potential of neuroblastoma. Proc Natl Acad Sci USA 105: 12891-12896, 2008.

4. Fleischmann A, Waser B and Reubi JC: Overexpression of gastrin-releasing peptide receptors in tumor-associated blood vessels of human ovarian neoplasms. Cell Oncol 29: 421-433, 2007.
5. Patel O, Shulkes A and Baldwin GS: Gastrin-releasing peptide and cancer. Biochim Biophys Acta 1766: 23-41, 2006.

6. Fan DM, Shi HR, Chen ZM, Wu QH, Liu HN and Zhang RT: Early detection of ovarian carcinoma by proteome profiling based on magnetic bead separation and matrix-assisted laser desorption/ionization time of flight mass spectrometry. African Journal of Microbiology Research 4: 940-951, 2010.

7. Patel O, Dumesny C, Giraud AS, et al: Stimulation of proliferation and migration of a colorectal cancer cell line by amidated and glycine-extended gastrin-releasing peptide via the same receptor. Biochem Pharmacol 68: 2129-2142, 2004.

8. Chinnappan D, Qu XP, Xiao DM, et al: Human gastrin-releasing peptide receptor gene regulation requires transcription factor binding at two distinct CRE sites. Am J Physiol Gastrointest Liver Physiol 295: G153-G162, 2008.

9. Schroeder RP, de Visser M, van Weerden WM, et al: Androgen-regulated gastrin-releasing peptide receptor expression in androgen-dependent human prostate tumor xenografts. Int J Cancer 126: 2826-2834, 2010.

10. Rassouli FB and Matin MM: Gene silencing in human embryonic stem cells by RNA interference. Biochem Biophys Res Commun 390: 1106-1110, 2009.

11. Zhu XL, Liang L and Ding YQ: Overexpression of FMNL2 is closely related to metastasis of colorectal cancer. Int J Colorectal Dis 23: 1041-1047, 2008.

12. Ischia J, Patel O, Shulkes A and Baldwin GS: Gastrin-releasing peptide: different forms, different functions. Biofactors 35: 69-75, 2009.

13. Qiao J, Kang J, Ishola TA, et al: Gastrin-releasing peptide receptor silencing suppresses the tumorigenesis and metastatic potential of neuroblastoma. Proc Natl Acad Sci USA 105: 12891-12896, 2008.

14. Liu X, Carlisle DL, Swick MC, et al: Gastrin-releasing peptide activates Akt through the epidermal growth factor receptor pathway and abrogates the effect of gefitinib. Exp Cell Res 313: 1361-1372, 2007

15. Ischia J, Patel O, Bolton D, Shulkes A and Baldwin GS: Expression and function of gastrin-releasing peptide (GRP) in normal and cancerous urological tissues. BJU Int 113 (Suppl 2): S40-S47, 2014

16. Paul P, Gillory LA, Kang J, Qiao J and Chung DH: Targeting gastrin-releasing peptide as a new approach to treat aggressive refractory neuroblastomas. Surgery 149: 425-432, 2011.

17. Lee S, Qiao J, Paul P and Chung DH: Integrin $\beta 1$ is critical for gastrin-releasing peptide receptor-mediated neuroblastoma cell migration and invasion. Surgery 154: 369-375, 2013.

18. Ni C, Zhao X, Sun T, Liu Y, Gu Q and Sun B: Role of gastrin-releasing peptides in breast cancer metastasis. Hum Pathol 43: 2342-2347, 2012. 